\title{
Integral input-to-state stability of interconnected iISS systems by means of a lower-dimensional comparison system
}

\author{
Björn S. Rüffer
}

\author{
Christopher M. Kellett
}

\author{
Steven R. Weller
}

\begin{abstract}
We consider arbitrarily many interconnected integral Input-to-State Stable (iISS) systems in an arbitrary interconnection topology and provide an (i)ISS comparison principle for networks. We show that global asymptotic stability of the origin (GAS) of a lower-dimensional system termed the comparison system, which is based on the individual dissipative Lyapunov iISS inequalities, together with a scaling condition implies the existence of an iISS Lyapunov function of the composite system. A sufficient (but not necessary) condition for 0-GAS of the interconnection is shown in this paper to be the generalized small-gain condition derived by Dashkovskiy $e t$ $a l$., but this time in a dissipative Lyapunov setting. We also provide geometric intuition behind growth rate conditions for the stability of cascaded iISS systems.

Index Terms-integral input-to-state stability, comparison principle, Lyapunov function, small-gain condition, large-scale systems
\end{abstract}

\section{INTRODUCTION}

Consider $n \geq 2$ control systems of the form

$$
\Sigma_{i}: \dot{x}_{i}=f_{i}\left(x_{1}, \ldots, x_{n}, u_{i}\right), \quad i=1, \ldots, n,
$$

satisfying dissipative integral input-to-state stability (iISS) estimates

$$
\begin{aligned}
\nabla V_{i}\left(x_{i}\right) \cdot f_{i}\left(x, u_{i}\right) \leq & -\alpha_{i}\left(V_{i}\left(x_{i}\right)\right)+\sum_{j \neq i} \gamma_{i j}\left(V_{j}\left(x_{j}\right)\right) \\
& +\gamma_{i u}\left(\left\|u_{i}\right\|\right),
\end{aligned}
$$

where each $V_{i}$ is assumed to be continuously differentiable, such that $\underline{\alpha}_{i}\left(x_{i}\right) \leq V_{i}\left(x_{i}\right) \leq \bar{\alpha}_{i}\left(x_{i}\right)$ for some $\mathcal{K}_{\infty}$ functions $\underline{\alpha}_{i}, \bar{\alpha}_{i}$, and the functions $\alpha_{i}, \gamma_{i j}, \gamma_{i u}$ are assumed to be locally Lipschitz continuous. The functions $\gamma_{i j}$ and $\gamma_{i u}$ are called gains and assumed to be of class $\mathcal{G}=\mathcal{K} \cup\{0\}$, i.e., they are each either $\mathcal{K}$ functions or zero. Throughout we assume that $\gamma_{i i}=0$. The functions $\alpha_{i}$ are assumed to be positive definite. If, in addition, the functions $\alpha_{i}$ are in class $\mathcal{K}_{\infty}$, then the systems $\Sigma_{i}$ are in fact input-to-state stable (ISS). It is known that an arbitrary composition of ISS systems is ISS, provided a small-gain condition is satisfied $[4,5]$.

B. S. Rüffer is with the Department of Electrical and Electronic Engineering, University of Melbourne, Parkville VIC 3010, Australia, phone +61-3-8344 0498, fax +61-3-8344 6678, bruef fer@unimelb. edu . au.

C. M. Kellett and S. R. Weller are with the School of Electrical Engineering and Computer Science, The University of Newcastle, Callaghan, NSW 2308, Australia, \{Chris.Kellett, Steven.Weller\}@newcastle.edu.au.

The authors have received support from the Australian Research Council (ARC) under grant DP0771131. This work has been submitted while the first author was based at the same institution as the other authors.
There exist numerous conditions in the literature $[2,3,6]$ for the stability of the composite system

$$
\Sigma: \dot{x}=f(x, u)
$$

arising by treating $\left(\Sigma_{1}, \ldots, \Sigma_{n}\right)$ as one single system under different forms of structural assumptions on the interconnection graph structure. Central to all existing results are growth and scaling conditions, which can be quite intricate.

In this paper we derive a lower-dimensional comparison system arising from the dissipation estimates (2) and show that stability properties of this comparison system carry over to the composite system (3). Moreover, we derive a smallgain condition that guarantees 0-GAS of the composite system. If in addition a Lyapunov function for the autonomous comparison system is known and satisfies a growth condition, then the composite system can be shown to be iISS. Our results apply to general interconnection topologies, which includes cascades and feedback cycles as special cases.

For the sake of a simpler exposition the right hand sides of estimates (2) (so-called supply rates) are not given in terms of norms of the states but in terms of Lyapunov functions of the states. This will ease notation dramatically. Moreover, we only treat the time-invariant case, but the time-varying case is a straightforward extension. Our approach is based on comparison techniques as detailed by Lakshmikantham and Leela $[10,11]$ and results on monotone dynamical systems [16] for the system induced by the interconnection topology, as well as a monotone selection theorem $[5,14]$ and converse Lyapunov theorems.

We use a nonlinear matrix-vector type formulation, by defining operators $A, \Gamma$ and $G: \mathbb{R}_{+}^{n} \rightarrow \mathbb{R}_{+}^{n}$ per $A(v)_{i}=$ $\alpha_{i}\left(v_{i}\right), \Gamma(v)_{i}=\sum_{j \neq i} \gamma_{i j}\left(v_{j}\right), G(w)_{i}=\gamma_{i u}\left(w_{i}\right), i=$ $1, \ldots, n$, and call

$$
\dot{v}=-A(v)+\Gamma(v)+G(w)
$$

the comparison system induced by the right hand sides of the dissipation inequalities (2). Associated is the autonomous system obtained by letting $w \equiv 0$,

$$
\dot{v}=M(v):=-A(v)+\Gamma(v) .
$$

Both systems are defined only for $v, w \in \mathbb{R}_{+}^{n}$. Using a converse theorem for the existence of a $\mathcal{C}^{1}$ Lyapunov function for (4)-(5), we use an appropriately scaled version of that Lyapunov function as a function of the vector $\left(V_{1}\left(x_{1}\right), \ldots, V_{n}\left(x_{n}\right)\right)^{T}$ as a candidate for a Lyapunov function for the composite system (3). 
As a sufficient condition for the existence of a Lyapunov function for (5) we derive a small-gain type condition of the form

$$
D \circ \tilde{\Gamma}(v) \nsupseteq v, \text { for all } v \in \mathbb{R}_{+}^{n}, v \neq 0,
$$

(where $¥$ denotes the logical negation, not the componentwise one, of the component-wise $\geq$ ordering). This condition exhibits considerable similarity with the general conditions stated in $[4,5,13]$ for input-to-state stability of arbitrary interconnections of ISS systems. In those papers condition (6) has been linked to GAS of an induced discrete-time system, whereas here it is linked to GAS of the continuoustime comparison system. In [4] ISS defined via trajectory estimates is considered, whereas in this paper we use a Lyapunov formulation and consider the more general class of integral ISS systems.

This paper is organized as follows: In Section II we establish some necessary notation. In Section III we define the comparison system induced by the dissipation inequalities (2) and state a few of its properties such as monotonicity of solutions. Section IV provides us with a condition for global asymptotic stability of the origin of the comparison system reminiscent of the generalized small-gain type conditions in $[4,5,13]$. In Section V we establish the main results of this paper, which are an iISS comparison principle and the construction of an iISS Lyapunov function for the composite system based on the assumption that the comparison system is iISS. We also present an example that provides a geometric interpretation of scaling conditions that are used in the literature. Section VI concludes the paper.

\section{PREliminaries}

In this section we settle some necessary notation. The positive orthant $\mathbb{R}_{+}^{n}$ in $\mathbb{R}^{n}$ is the set $\left\{x \in \mathbb{R}^{n}: x_{i} \geq 0 \forall i\right\}$. By the boundary of $\mathbb{R}_{+}^{n}$, also denoted $\partial \mathbb{R}_{+}^{n}$, we mean the set $\left\{s \in \mathbb{R}_{+}^{n}: \exists i: s_{i}=0\right\}$. In $\mathbb{R}^{n}$ the open ball of radius $r>0$ centered at $x$ is denoted by $B(x, r)$. Sometimes we use an open ball in $\mathbb{R}_{+}^{n}$, and then it is understood that this really is $B(x, r) \cap \mathbb{R}_{+}^{n}$. The $p$-norm on $\mathbb{R}^{n}$ is denoted by $\|\cdot\|_{p}$, where $p$ is usually omitted in the case $p=2$. The max-norm is denoted as $\|\cdot\|_{\infty}$. The sphere with respect to the one norm, intersected with the positive orthant $\mathbb{R}_{+}^{n}$ is an $(n-1)$-simplex, and denoted by

$$
S_{r}:=\left\{x \in \mathbb{R}_{+}^{n}:\|x\|_{1}=r\right\} .
$$

The comparison function classes $\mathcal{K}$ and $\mathcal{K}_{\infty}$ are, respectively, the sets of continuous functions $\left\{\gamma: \mathbb{R}_{+} \rightarrow\right.$ $\mathbb{R}_{+}, \gamma(0)=0, \gamma$ is strictly increasing $\}$ and $\{\gamma \in \mathcal{K}$ : $\gamma$ is unbounded $\}$.

The order on $\mathbb{R}^{n}$ is given by $x \leq y$ if and only if $x_{i} \leq y_{i}$ for all $i$; $x<y$ if and only if $x \leq y$ and $x \neq y$; and $x \ll y$ if and only if $x_{i}<y_{i}$ for all $i$. Notably, the condition $x \nsupseteq y$ is not the same as $x<y$ but denotes the following statement:

"There exists at least one component $i$, such that

$x_{i}<y_{i}$."

In other words, $x \nsupseteq y$ means: Either $x<y$ or $x$ and $y$ are not comparable.

\section{STABILITY OF THE COMPARISON SYSTEM}

Consider the comparison system (5) arising from (2), where $M$ is a (nonlinear) Metzler operator defined by $M=$ $-A+\Gamma$, i.e.,

$$
(M(v))_{i}=-\alpha_{i}\left(v_{i}\right)+\sum_{j \neq i} \gamma_{i j}\left(v_{j}\right) .
$$

Throughout we assume that the functions $\alpha_{i}, \gamma_{i j}, \gamma_{i u}$ are locally Lipschitz, guaranteeing existence and uniqueness of solutions for (5) and also for the applicability of converse Lyapunov theorems. We denote solutions of (5) by $\phi$ : $\mathbb{R}_{+} \times \mathbb{R}^{n} \rightarrow \mathbb{R}^{n}$, i.e., a solution of (5) at time $t \geq 0$ from an initial condition $v \in \mathbb{R}^{n}$ is denoted by $\phi(t, v)$.

A Metzler operator is by definition quasimonotone nondecreasing (cf. Lakshmikantham and Leela, [12]) which is the same as type $K$ (cf. Smith, [16]), i.e., for each $i$, $M(v)_{i} \leq M(u)_{i}$ for any points $v$ and $u$ that satisfy $v \leq u$ and $v_{i}=u_{i}$. Observe that the origin is an equilibrium point of (5).

Lemma III.1 System (5) is positive, i.e., solutions starting in the positive orthant $\mathbb{R}_{+}^{n}$ evolve, as long as they exist, in this orthant.

Proof. It suffices to consider what happens to trajectories hitting the boundaries of $\mathbb{R}_{+}^{n}$. Let $\phi(\cdot)$ denote a solution and assume $\phi(t)=b$, where $b_{i}=0$ for at least one $i$, i.e., the solution hits the boundary of $\mathbb{R}_{+}^{n}$ at time $t$.

For each $i$ such that $b_{i}=0$ we have for the $i$ th component of $\left.\frac{d}{d s}\right|_{s=t} \phi(s)=M b$ that $(M b)_{i}=-\alpha_{i}(0)+$ $\sum_{j \neq i} \gamma_{i j}\left(b_{j}\right) \geq 0$. This shows that the positive orthant is invariant for system (5).

Global asymptotic stability of the origin with respect to system (5) (for short we say (5) is GAS) is defined as

1) all trajectories converge to the origin (global attractivity) and

2) the origin is stable in the sense of Lyapunov.

System (3) is 0-GAS, if the origin is GAS for (3) when $u \equiv 0$, cf. [18].

The comparison principle [10, Theorem 4.1.2, p.268] states that stability properties of the trivial solution of (5) carry over to the trivial solution of system (3):

Proposition III.2 (Comparison principle) If system (5) is GAS, then system (3) is $0-G A S$.

Another important fact regarding solutions of the comparison system (5) concerns the ordering of solutions. We have the following due to [16, Proposition 1.1, p.32]:

Proposition III.3 (Ordering of solutions) Let $u^{0}, v^{0} \in \mathbb{R}_{+}^{n}$, then on the maximal interval $J=[0, T)$ where both solutions of (5) exist, the following implications hold for $t \in J$,

1) if $u^{0} \leq v^{0}$ then $\phi\left(t, u^{0}\right) \leq \phi\left(t, v^{0}\right)$;

2) if $u^{0}<v^{0}$ then $\phi\left(t, u^{0}\right)<\phi\left(t, v^{0}\right)$; and

3) if $u^{0} \ll v^{0}$ then $\phi\left(t, u^{0}\right) \ll \phi\left(t, v^{0}\right)$.

Scaling and growth conditions on the supply rates in [3] and [6] turn out to be closely connected to the concept of 
decay sets [14]. We define the $i$ th decay set to be

$$
\Omega_{i}:=\left\{v \in \mathbb{R}_{+}^{n}:(M(v))_{i}<0\right\} .
$$

This is the domain where trajectories of the comparison system (5) decrease in their $i$ th component. The next result shows that - provided that the origin is globally attractiveevery trajectory with respect to (5) has to be in one of the $\Omega_{i}$ sets at any given time. In other words,

$$
\bigcup_{i=1}^{n} \Omega_{i}=\mathbb{R}_{+}^{n} \backslash\{0\} .
$$

Proposition III.4 If the origin is globally attractive with respect to (5), then the Metzler operator $M$ satisfies

$$
M(v) \nsupseteq 0, \quad \forall v \in \mathbb{R}_{+}^{n}, v \neq 0 .
$$

Proof. We argue by contradiction. Suppose there exists $v^{0}>$ 0 , such that $M\left(v^{0}\right) \geq 0$. Firstly, $M\left(v^{0}\right)=0$ implies the existence of an equilibrium at $v^{0}$, contradicting global attractivity of the origin. So we have $M\left(v^{0}\right)>0$. By global attractivity of the origin system (5) is forward complete. So there exists an $\varepsilon>0$ such that $\phi\left(t, v^{0}\right)>v^{0}$ for all $0<t \leq \varepsilon$. Denote $v^{1}:=\phi\left(\varepsilon, v^{0}\right)>v^{0}$.

Now let $u^{0}>v^{0}$. Using the ordering of solutions, Proposition III.3, we have $\phi\left(t, u^{0}\right)>\phi\left(t, v^{0}\right)>v^{0}$ for all $0<t \leq \varepsilon$. Denoting $u^{1}:=\phi\left(\varepsilon, u^{0}\right)>v^{1}>v^{0}$, we find $\phi\left(t+\varepsilon, u^{0}\right)=\phi\left(t, u^{1}\right)>u^{1}>u^{0}$ for $0<t \leq \varepsilon$. Repeating the argument we obtain

$$
\phi\left(t, u^{0}\right)>u^{0}, \quad \forall t>0,
$$

contradicting global attractivity of the origin. This shows that there cannot exist $v^{0} \in \mathbb{R}_{+}^{n}, v^{0} \neq 0$, such that $M\left(v^{0}\right) \geq 0$. In other words, $M(v) \nsupseteq 0$ for all $v \in \mathbb{R}_{+}^{n}, v \neq 0$.

In $[4,5]$ a condition similar to $(8)$ has been recognized as a general small-gain type condition, guaranteeing stability of interconnections of ISS systems. We have the following result, which is based on the Knaster-KuratowskiMazurkiewicz (KKM) principle:

Theorem III.5 Assume $M$ is such that (8) holds. Then for each $r>0$ there exists $a v \in \mathbb{R}_{+}^{n}, v \gg 0,\|v\|=r$, such that $M(v) \ll 0$. In other words, for all $r>0$,

$$
\bigcap_{i=1}^{n} \Omega_{i} \cap S_{r} \neq \emptyset \text {. }
$$

The proof of this result is essentially the same as that of the corresponding result in [4].

The previous result states that there exists a decay set,

$$
\Omega:=\bigcap_{i=1}^{n} \Omega_{i}
$$

along which solutions decrease in all components, provided that the origin is globally attractive. An interesting consequence is the following (cf. [14, Prop. 4.2] for a discrete-time version):

Lemma III.6 For a monotone system as in (5) the origin is stable if it is attractive.
Proof. For every $\varepsilon>0$ there exists a $\delta>0$ such that the open ball $B_{\delta}(0)<S_{\varepsilon} \cap \Omega$ and solutions starting in $B_{\delta}(0)$ will remain in $B_{\varepsilon}(0)<S_{\varepsilon}$ by monotonicity, due to Prop. III.3.

\section{SMALL-GAIN CONDITION}

Here we provide a small-gain condition guaranteeing GAS of the comparison system.

Theorem IV.1 Consider the comparison system (5) with operators $\Gamma, A: \mathbb{R}_{+}^{n} \rightarrow \mathbb{R}_{+}^{n}$, where we assume $\gamma_{i j} \in$ $\mathcal{K}_{\infty} \cup\{0\}$. If there exist diagonal operators

- $T: \mathbb{R}_{+}^{n} \rightarrow \mathbb{R}_{+}^{n}, T(v)_{i}=\tau_{i}\left(v_{i}\right), \tau_{i} \in \mathcal{K}_{\infty}$ satisfying $\tau_{i}+\alpha_{i} \in \mathcal{K}_{\infty}$ and

- $D: \mathbb{R}_{+}^{n} \rightarrow \mathbb{R}_{+}^{n}, D(v)_{i}=v_{i}+\delta_{i}\left(v_{i}\right), \delta_{i} \in \mathcal{K}_{\infty}$,

such that

$$
D \circ(\Gamma+T) \circ(T+A)^{-1}(v) \nsupseteq v, \quad \forall v>0,
$$

then the origin is GAS with respect to system (5).

By application of Prop. III.2 the small-gain condition implies 0-GAS of the network of iISS systems:

Corollary IV.2 Assume $n \geq 2$ interconnected systems (1) are given together with iISS Lyapunov functions $V_{i}$ satisfying (2), $i=1, \ldots, n$. Then under the assumptions of Theorem IV.1 the composite system (3) is 0-GAS.

Note that $(T+A)$ is a diagonal operator with $\mathcal{K}_{\infty}$ entries on the diagonal, so its inverse is of the same shape. Moreover the composite operator $\tilde{\Gamma}:=(\Gamma+T) \circ(T+A)^{-1}$ is of the form $\tilde{\Gamma}(v)_{i}=\sum_{j} \tilde{\gamma}_{i j}\left(v_{j}\right)$, where

$$
\tilde{\gamma}_{i j}= \begin{cases}\tau_{i} \circ\left(\tau_{i}+\alpha_{i}\right)^{-1} & \text { if } j=i, \\ \gamma_{i j} \circ\left(\tau_{j}+\alpha_{j}\right)^{-1} & \text { otherwise, }\end{cases}
$$

and $\tilde{\gamma}_{i j}$ is of class $\mathcal{K}_{\infty}$ for $i=j$, and of class $\mathcal{K}_{\infty} \cup\{0\}$ otherwise.

Remark IV.3 Given a locally Lipschitz continuous, positive definite function $\alpha: \mathbb{R}_{+} \rightarrow \mathbb{R}_{+}$, there always exists a function $\tau \in \mathcal{K}_{\infty}$, such that $\alpha+\tau \in \mathcal{K}_{\infty}$. To see this, simply note that the right hand side derivative $D^{+} \alpha(r):=$ $\lim _{h \rightarrow 0^{+}} \frac{\alpha(r+h)-\alpha(r)}{h}$ exists for all $r \geq 0$ and is bounded on compact intervals. So for arbitrary small $\varepsilon>0$ we might take

$$
\tau(r):=\int_{0}^{r} \varepsilon+\max \left\{0,-D^{+} \alpha(s)\right\} d s
$$

so that $D^{+}(\alpha+\tau)(r)>0$ for all $r \geq 0$.

Consequently, the hard part in Theorem IV.1 is to find a suitable operator $D$ and to check the general small-gain condition, which is essentially the same task that is required in $[4,5]$ for the interconnection stability of ISS systems.

Sketch of the proof of Theorem IV.1. It suffices to note that (10), or equivalently, $D \circ \tilde{\Gamma} \nsucceq$ id implies the existence of a component-wise unbounded path $\sigma$ in $\mathbb{R}_{+}^{n}$, parametrized by $\mathcal{K}_{\infty}$ functions $\sigma_{i}$ such that $\tilde{\Gamma}(\sigma(r)) \ll \sigma(r)$ for all $r>0$, cf. [5, 13,14]. It is important that (10) in particular implies $(-A+\Gamma)(\tilde{\sigma}) \ll 0$, with $\tilde{\sigma}_{i}=\left(\tau_{i}+\alpha_{i}\right)^{-1} \circ \sigma_{i} \in \mathcal{K}_{\infty}$. Hence we have $\Omega \cap S_{r} \neq 0$ for all $r>0$. 
Now for every $v \in \mathbb{R}_{+}^{n}$ there exists an $r^{v}>0$ such that $\tilde{\sigma}\left(r^{v}\right) \geq v$. Since $\tilde{\sigma}\left(r^{v}\right) \in \Omega$ and $\Omega$ can be shown to be invariant, $\phi\left(t, \tilde{\sigma}\left(r^{v}\right)\right)$ exists for all $t \geq 0$ and hence $0 \leq$ $\phi(t, v) \leq \phi\left(t, \tilde{\sigma}\left(r^{v}\right)\right)$ for all $t \geq 0$ such that $\phi(t, v)$ exists. This implies the existence of $\phi(t, v)$ for all $t \geq 0$ and also that $\lim _{t \rightarrow \infty} \phi(t, v)=0$, proving attractivity of the origin. Stability of the origin follows from Lemma III.6.

Remark IV.4 The small-gain condition as stated above is actually quite strong, as it does rule out true iISS systems that are not ISS:

As we have seen in the previous proof, condition (10) implies the existence of a $\mathcal{K}_{\infty}^{n}$ path $\tilde{\sigma}$, such that $(-A+$ $\Gamma)(\tilde{\sigma}) \ll 0$. For each $i=1, \ldots, n$ and $r>0$ this implies

$$
\alpha_{i}\left(\tilde{\sigma}_{i}(r)\right)>\sum_{i j} \gamma_{i j}\left(\tilde{\sigma}_{j}(r)\right) .
$$

If a subsystem has no inputs, then it is GAS and hence ISS. If it has inputs then at least one $\gamma_{i j} \in \mathcal{K}_{\infty}$ and the above inequality implies $\alpha_{i}(r) \rightarrow \infty$ as $r \rightarrow \infty$. Therefore $\alpha_{i}$ can be bounded from below by a $\mathcal{K}_{\infty}$ function, which implies that the corresponding system $\Sigma_{i}$ is ISS.

Remark IV.5 (Cascade of a GAS and an iISS system) For the cascade of two systems

$$
\begin{aligned}
& \Sigma_{1}: \dot{x}=f(x, z) \\
& \Sigma_{2}: \dot{z}=g(z),
\end{aligned}
$$

where the origin is GAS with respect to $\Sigma_{2}$, and $\Sigma_{1}$ is assumed iISS (cf. [2,6]), we have Lyapunov functions $V_{i}$, positive definite $\alpha_{i}$, and $\gamma \in \mathcal{K}_{\infty}$ satisfying

$$
\nabla V_{1}(x) f(x, z) \leq-\alpha_{1}\left(V_{1}(x)\right)+\gamma\left(V_{2}(z)\right)
$$

and

$$
\nabla V_{2}(z) g(z) \leq-\alpha_{2}\left(V_{2}(z)\right)
$$

The sets $\Omega_{i}$ can take the form as depicted in Figure 1, where $\Omega_{2}=\left\{v \in \mathbb{R}_{+}^{2}: 0<-\alpha_{2}\left(v_{2}\right)\right\}=\mathbb{R}_{+}^{2} \backslash\left\{v: v_{2}=0\right\}$, and $\Omega_{1}=\left\{v: 0<-\alpha_{1}\left(v_{1}\right)+\gamma\left(v_{2}\right)\right\}=\left\{v \in \mathbb{R}_{+}^{2}: v_{2}<\right.$ $\left.\left(\gamma^{-1} \circ \alpha_{1}\right)\left(v_{1}\right)\right\}$. Figure 1 indicates that the $v_{2}$-dynamics have to be faster than the $v_{1}$ dynamics, so that solutions of the comparison system cannot escape to the right in Figure 1, but instead eventually enter the set $\Omega=\Omega_{1} \cap \Omega_{2}$.

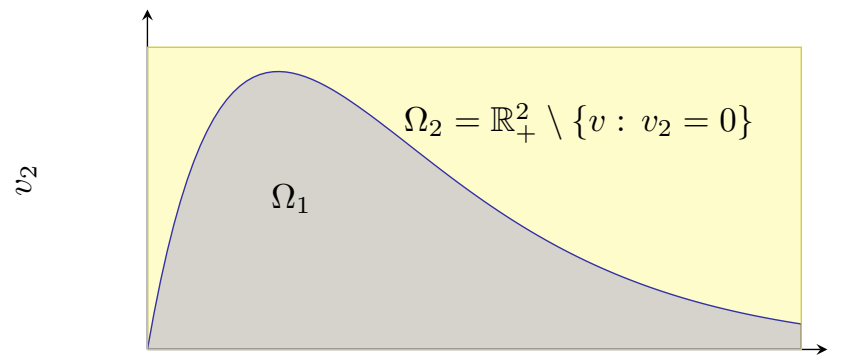

Fig. 1. Decay sets might asymptotically cling to the coordinate axis.
Remark IV.6 (Feedback interconnection of two ISS systems) For two feedback interconnected systems of the form

$$
\begin{aligned}
& \Sigma_{1}: \dot{x}=f(x, z) \\
& \Sigma_{2}: \dot{z}=g(z, x),
\end{aligned}
$$

which are both ISS, the small-gain condition (cf. Theorem IV.1, [8,9]) ensures a particular shape of the set $\Omega=$ $\Omega_{1} \cap \Omega_{2}$, so that an unbounded $\mathcal{K}_{\infty}^{n}$ path can evolve in it, cf. Figure 2.

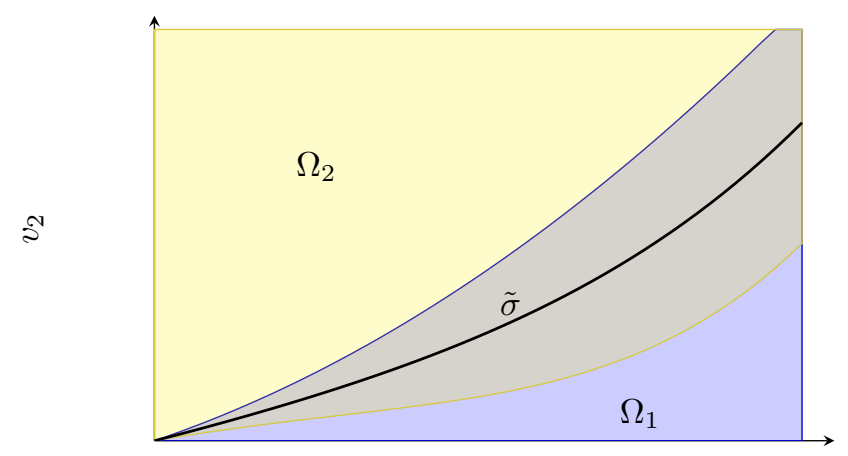

Fig. 2. If there exists a $\mathcal{K}_{\infty}^{n}$ path $\tilde{\sigma}$ such that $(-A+\Gamma)(\tilde{\sigma}) \ll 0$, then the decay sets cannot asymptotically cling to the coordinate axis.

Theorem III.5 suggests that the condition (8) would be a good candidate for a small-gain condition. Unfortunately, (8) alone is too weak to imply GAS of the origin with respect to (5). It is not too difficult to construct a simple cascade of two nonlinear systems, such that the resulting Metzler operator satisfies $M \nsupseteq \mathrm{id}$, and yet the origin is not GAS.

But still we can draw interpretation from condition (8). Looking at Figure 1, we know that $\Omega$ is unbounded, but far away from the origin it might cling to an axis.

Scaling conditions as provided in [3] can ensure that $\Omega$ is reached in finite time. For feed-forward structures these conditions are easy to formulate. A relatively simple one is that the decay rate $-\alpha_{j}$ of the driving system has to be greater than the gain $\gamma_{i j}$ of the driven system in a neighborhood of the origin, i.e., that $\limsup _{h \rightarrow 0^{+}} \frac{\gamma_{i j}(h)}{\alpha_{j}(h)}$ is finite, cf. [3], where also more involved conditions are stated.

\section{INTEGRAL INPUT-TO-STATE STABILITY OF THE COMPOSITE SYSTEM}

The first result in this section is an iISS comparison principle, translating stability properties of the comparison system into stability properties of the composite system (3). This result is more general than existing stability results for cascades and feedback interconnections as it applies to arbitrary interconnection topologies of subsystems that are possibly only iISS but not ISS. Yet it is known that a feedback loop of only two iISS systems, under a small-gain condition, is iISS only if one of the systems is at least ISS, cf. [6]. These types of restrictions are hidden in the stability assumption of the comparison system. 
Theorem V.1 (An (i)ISS comparison principle) Assume $n \geq$ 2 interconnected systems (1) are given together with iISS Lyapunov functions $V_{i}$ satisfying (2), $i=1, \ldots, n$.

Then (integral) input-to-state stability of the comparison system (4) from $w$ to $v$ implies the same corresponding stability property for the system (3).

Moreover, if the smooth (integral) ISS Lyapunov function for (14) is denoted by $L$, then the corresponding (integral) ISS Lyapunov function for the nominal system can be taken as $V(x)=L\left(\left(V_{1}\left(x_{1}\right), \ldots, V_{n}\left(x_{n}\right)\right)^{T}\right)$.

Proof. Rewriting the prerequisites in vector notation and denoting

$$
\underline{V}(x):=\left(V_{1}\left(x_{1}\right), \ldots, V_{n}\left(x_{n}\right)\right)^{T}
$$

we have along trajectories $x(t)$ of (3) for the derivative of $\underline{V}$,

$$
\begin{aligned}
\frac{d}{d t}[\underline{V}(x(t))]= & \left(\left\langle\nabla V_{1}\left(x_{1}(t)\right), f_{1}\left(x, u_{1}\right)\right\rangle, \ldots\right. \\
& \left.\ldots,\left\langle\nabla V_{n}\left(x_{n}(t)\right), f_{n}\left(x, u_{n}\right)\right\rangle\right)^{T} \\
\leq & M(\underline{V}(x(t)))+G\left(\left(\left\|u_{1}\right\|, \ldots,\left\|u_{n}\right\|\right)^{T}\right) .
\end{aligned}
$$

By assumption there exists a smooth function $L: \mathbb{R}_{+}^{n} \rightarrow$ $\mathbb{R}_{+}$, due to an ISS (respectively, iISS) converse Lyapunov theorem, see [17], resp. [1], such that there exist two class $\mathcal{K}_{\infty}$ functions $\underline{\alpha}, \bar{\alpha}$, so that

$$
\underline{\alpha}(\|v\|) \leq L(v) \leq \bar{\alpha}(\|v\|), \quad \text { for all } v \in \mathbb{R}_{+}^{n},
$$

and there exist a positive definite function $\alpha\left(\alpha \in \mathcal{K}_{\infty}\right.$ in the ISS case) and $\gamma \in \mathcal{K}$ such that for all $v, w \in \mathbb{R}_{+}^{n}$,

$$
\langle\nabla L(v), M(v)+G(w)\rangle \leq-\alpha(\|v\|)+\gamma(\|w\|) .
$$

Now define $V(x):=L(\underline{V}(x))$. Then we have

$$
\begin{aligned}
& \langle\nabla V(x), f(x, u)\rangle=\langle\nabla L(\underline{V}(x)), \\
& \left.\left.\left(\left\langle\nabla V_{1}\left(x_{1}\right), f_{1}\left(x, u_{1}\right)\right\rangle, \ldots \ldots, \nabla V_{n}\left(x_{n}\right), f_{n}\left(x, u_{n}\right)\right\rangle\right)^{T}\right\rangle \\
\leq & \langle\nabla L(\underline{V}(x)), M(\underline{V}(x))+G(u)\rangle \leq-\alpha(\|\underline{V}(x)\|)+\gamma(\|u\|) .
\end{aligned}
$$

Using that $V_{i}\left(x_{i}\right) \geq \underline{\alpha}_{i}\left(\left\|x_{i}\right\|\right)$, it is clear that the last inequality implies a dissipative (integral) ISS estimate with smooth (i)ISS Lyapunov function $V=L \circ \underline{V}$.

The next result combines a weaker stability assumption for the comparison system with a scaling condition to deduce iISS of the composite system (3).

Theorem V.2 Assume $n \geq 2$ interconnected systems (1) are given together with iISS Lyapunov functions $V_{i}$ satisfying (2), $i=1, \ldots, n$. Then the composite system (3) is $0-G A S$ if the origin is GAS with respect to the comparison system (5). If in addition

$$
\varphi(r):=\int_{0}^{r} \frac{1}{\max _{v: L(v)=s}\|\nabla L(v)\|_{\infty}} d s,
$$

is unbounded, where $L$ is a Lyapunov function for system (5), then the composite system (3) is integral input-to-state stable with respect to $u$.
Proof of Theorem V.2. Since the origin is GAS with respect to (5), there exists a $\mathcal{C}^{1}$ Lyapunov function $L: \mathbb{R}_{+}^{n} \rightarrow \mathbb{R}_{+}$, and functions $\underline{\alpha}, \bar{\alpha} \in \mathcal{K}_{\infty}$, such that $\underline{\alpha}(\|v\|) \leq L(v) \leq$ $\bar{\alpha}(\|v\|)$ and $\nabla L(v) \cdot M(v)<0$ for all $v>0$, cf. e.g. [19] for an appropriate converse theorem ${ }^{1}$.

Since the level sets $\left\{v \in \mathbb{R}_{+}^{n}: L(v)=r\right\}$ are compact, $\nabla L$ is continuous, and by $\nabla L(v) \cdot M(v) \neq 0$ for all $v>0$, we have that the function $\varphi: \mathbb{R}_{+}^{n} \rightarrow \mathbb{R}_{+}^{n}$ defined by (11) is a continuously differentiable and strictly increasing function. By assumption it is also unbounded, hence of class $\mathcal{K}_{\infty}$.

Now consider the Lyapunov function candidate $V$ for the composite system (3) given by

$$
V(x):=\varphi \circ L\left(\left(V_{1}\left(x_{1}\right), \ldots, V_{n}\left(x_{n}\right)\right)^{T}\right) .
$$

This candidate is bounded from below and above by class $\mathcal{K}_{\infty}$ functions: $V(x) \geq \varphi \circ$ $\underline{\alpha}\left(\left\|\underline{\alpha}_{1}\left(\left\|x_{1}\right\|\right), \ldots, \underline{\alpha}_{n}\left(\left\|x_{n}\right\|\right)\right\|\right) \geq \underline{\underline{\alpha}}(\|x\|)$ for some $\underline{\underline{\alpha}} \in \mathcal{K}_{\infty}$ and similarly $V(x) \leq \overline{\bar{\alpha}}(\|x\|)$ for some $\overline{\bar{\alpha}} \in \mathcal{K}_{\infty}$.

Denoting the $i$ th unit vector in $\mathbb{R}^{n}$ by $e_{i}$, the derivative of $V$ along trajectories of (3) is given by

$$
\begin{aligned}
& \nabla V(x) \cdot f(x, u)= \\
& \varphi^{\prime}\left(L\left(\left(V_{1}\left(x_{1}\right), \ldots, V_{n}\left(x_{n}\right)\right)^{T}\right)\right) . \\
& \sum_{i=1}^{n} \frac{\partial L}{\partial v_{i}}\left(\left(V_{1}\left(x_{1}\right), \ldots, V_{n}\left(x_{n}\right)\right)^{T}\right) \cdot \nabla V_{i}\left(x_{i}\right) \cdot f_{i}\left(x, u_{i}\right) \\
& \leq \varphi^{\prime}\left(L\left(\left(V_{1}\left(x_{1}\right), \ldots, V_{n}\left(x_{n}\right)\right)^{T}\right)\right) . \\
& \nabla L\left(\left(V_{1}\left(x_{1}\right), \ldots, V_{n}\left(x_{n}\right)\right)^{T}\right) . \\
& {\left[M\left(\left(V_{1}\left(x_{1}\right), \ldots, V_{n}\left(x_{n}\right)\right)^{T}\right)+\sum_{i} \gamma_{i u}\left(\left\|u_{i}\right\|\right) \cdot e_{i}\right]} \\
& \leq-\alpha(V(x))+\max _{i} \gamma_{i u}\left(\left\|u_{i}\right\|\right)
\end{aligned}
$$

where we have used the fact that $\left\|\varphi^{\prime}(L(v)) \cdot \nabla L(v)\right\|_{\infty} \leq 1$, and took $\alpha: \mathbb{R}_{+} \rightarrow \mathbb{R}_{+}$to be the positive definite function given by

$$
\alpha(s):=\min _{v: \varphi \circ L(v)=s}\left(-\varphi^{\prime}(L(v)) \nabla L(v) \cdot M(v)\right) .
$$

Clearly (12) is an iISS estimate for the composite system (3), which completes the proof.

Lastly, we discuss an example application of our result to give at least an indication how it compares to the recent results in [7], from where we borrowed the following cascaded systems:

Example V.3 Consider the cascade of an iISS system (13) driven by a GAS system (14), given by

$$
\begin{aligned}
& \dot{x}_{1}=-x_{1}+x_{1} x_{2} \\
& \dot{x}_{1}=-x_{2}^{3}
\end{aligned}
$$

with Lyapunov functions $V_{1}\left(x_{1}\right)=\frac{1}{2} \log \left(x_{1}^{2}+1\right)$ and $V_{2}\left(x_{2}\right)=\frac{1}{2} x_{2}^{2}$. Utilizing the computations in [7], and

\footnotetext{
${ }^{1}$ As one reviewer pointed out, the cited result has been derived for the case when the basin of attraction is the whole of $\mathbb{R}^{n}$, not just one orthant. However, the positive orthant is invariant with respect to our comparison system (Lemma III.1) and due to local Lipschitz continuity of $M$, GAS is robust. Hence the same construction also works in our case.
} 
expressing dissipation estimates in terms of Lyapunov functions, we obtain

$$
\begin{aligned}
& \dot{V}_{1} \leq-\frac{e^{2 V_{1}\left(x_{1}\right)}-1}{e^{2 V_{1}\left(x_{1}\right)}}+\sqrt{2 V_{2}\left(x_{2}\right)} \\
& \dot{V}_{2}=-4\left(V_{2}\left(x_{2}\right)\right)^{2} .
\end{aligned}
$$

The driven system (13) is only iISS and not ISS, as the term $\alpha_{1}\left(v_{1}\right)=\frac{e^{2 v_{1}}-1}{e^{2 v_{1}}}$ is only positive definite and not of class $\mathcal{K}_{\infty}$. Further we have $\alpha_{2}\left(v_{2}\right)=-4 v_{2}^{2}$ and $\gamma_{12}\left(v_{2}\right)=$ $\sqrt{2} \sqrt{v_{2}}$. This leads us to the Metzler system

$$
\dot{v}=M(v)=\left[\begin{array}{c}
-\alpha_{1}\left(v_{1}\right)+\gamma_{12}\left(v_{2}\right) \\
-\alpha_{2}\left(v_{2}\right)
\end{array}\right] .
$$

The arrangement of the sets $\Omega_{i}$ is depicted in Figure 3, i.e., for large $v_{1}$ cross-section of $\Omega_{1}$ contain almost all of the interval $\left[0, \frac{1}{2}\right)$. The set $\Omega_{2}$ contains the interior of $\mathbb{R}_{+}^{2}$. Since for $v>0$ either $v_{2}>0$ and hence $M(v)_{2}<0$ or $v_{2}=0$ so $M(v)_{1}<0$, the intersection $\Omega=\Omega_{1} \cap \Omega_{2}$ has to be radially nonempty by Theorem III.5.

Observe that the $v_{2}$-dynamics will reach any compact interval $[0, \varepsilon], \varepsilon>0$, in finite time, while the existence of solutions to the $v_{1}$-dynamics is guaranteed by comparing it's dynamics with

$$
\dot{v}_{1} \leq-\alpha_{1}\left(v_{1}\right)+\gamma\left(v_{2}^{0}\right) \leq \gamma\left(v_{2}^{0}\right)=\text { const },
$$

i.e., $v_{2}$ can grow at most linearly with time. Together this implies that every nontrivial trajectory with respect to (17) eventually enters the set $\Omega$, and from here GAS follows easily.

Hence there exists a Lyapunov function $L$ for the comparison system (17) and we can proceed as in the proof of Theorem V.2 to construct a Lyapunov function $V$ for the original cascade (13)-(14).

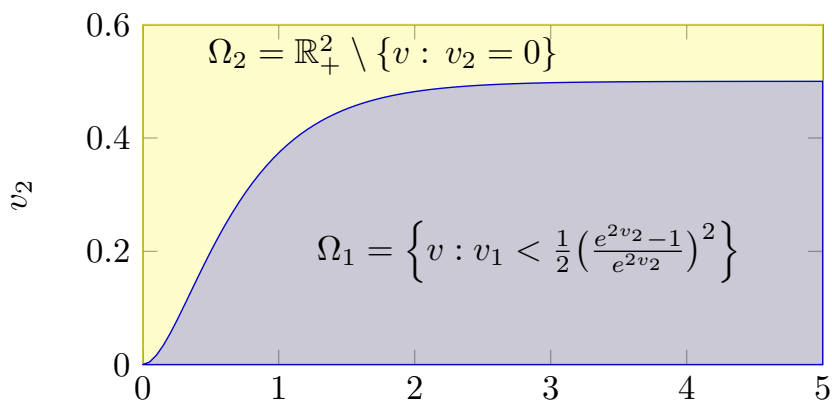

Fig. 3. The decay set in Example V.3 is reached by the $v_{2}$-dynamics in finite time.

While the previous example sheds some light on the cascade growth conditions used in $[3,6,7]$, the procedure of comparing decrease rates is not quite applicable for interconnection structures containing loops. More advanced considerations to ensure GAS for the comparison system have to be found for this case.

\section{CONCLUSions}

In this paper we have aggregated given iISS Lyapunov functions of subsystems to one iISS Lyapunov function for the composite system, provided that an associated comparison system is iISS. We have shown that the general ISS small-gain condition, which is known from trajectory-based and implication-Lyapunov formulations, has its counterpart in the dissipative Lyapunov formulation. In this case the validity of the ISS small-gain condition in fact implies that each of the interconnected subsystems has to be ISS.

\section{REFERENCES}

[1] D. Angeli, E. D. Sontag, and Y. Wang, A characterization of integral input-to-state stability, IEEE Trans. Automat. Control 45 (2000), no. 6, 1082-1097.

[2] M. Arcak, D. Angeli, and E. D. Sontag, A unifying integral ISS framework for stability of nonlinear cascades, SIAM J. Control Optim. 40 (2002), no. 6, 1888-1904 (electronic).

[3] A. Chaillet and D. Angeli, Integral input to state stable systems in cascade, Systems Control Lett. 57 (2008), no. 7, 519-527.

[4] S. N. Dashkovskiy, B. S. Rüffer, and F. R. Wirth, An ISS smallgain theorem for general networks, Math. Control Signals Systems 19 (2007), no. 2, 93-122.

[5] Small gain theorems for large scale systems and construction of ISS Lyapunov functions (2009). Submitted, available online at http://www.sigpromu.org.

[6] H. Ito, State-dependent scaling problems and stability of interconnected iISS and ISS systems, IEEE Trans. Automat. Control 51 (2006), no. $10,1626-1643$.

[7] A Lyapunov Approach to Integral Input-to-State Stability of Cascaded Systems with External Signals, Proc. of the 47th IEEE Conference on Decision and Control, CDC 2008, 2008, Dec. 9, pp. 628-633.

[8] Z.-P. Jiang, I. M. Y. Mareels, and Y. Wang, A Lyapunov formulation of the nonlinear small-gain theorem for interconnected ISS systems, Automatica J. IFAC 32 (1996), no. 8, 1211-1215.

[9] Z.-P. Jiang, A. R. Teel, and L. Praly, Small-gain theorem for ISS systems and applications, Math. Control Signals Systems 7 (1994), no. 2, 95-120.

[10] V. Lakshmikantham and S. Leela, Differential and integral inequalities: Theory and applications. Vol. I: Ordinary differential equations, Academic Press, New York, 1969. Mathematics in Science and Engineering, Vol. 55-I.

[11] Differential and integral inequalities: Theory and applications. Vol. II: Functional, partial, abstract, and complex differential equations, Academic Press, New York, 1969. Mathematics in Science and Engineering, Vol. 55-II.

[12] V. Lakshmikantham, V. M. Matrosov, and S. Sivasundaram, Vector Lyapunov functions and stability analysis of nonlinear systems, Mathematics and its Applications, vol. 63, Kluwer Academic Publishers Group, Dordrecht, 1991.

[13] B. S. Rüffer, Monotone dynamical systems, graphs, and stability of large-scale interconnected systems, Ph.D. Thesis, 2007. Available online at http://nbn-resolving.de/urn:nbn:de:gbv:46-diss000109058.

[14] __ Monotone inequalities, dynamical systems, and paths in the positive orthant of Euclidean n-space, Positivity (2009). To appear, DOI 10.1007/s11117-009-0016-5.

[15] B. S. Rüffer, C. M. Kellett, and S. R. Weller, Connection between cooperative positive systems and integral input-to-state stability of large-scale systems. Submitted, 2009.

[16] H. L. Smith, Monotone dynamical systems, Mathematical Surveys and Monographs, vol. 41, American Mathematical Society, Providence, RI, 1995. An introduction to the theory of competitive and cooperative systems.

[17] E. D. Sontag and Y. Wang, On characterizations of the input-to-state stability property, Systems Control Lett. 24 (1995), no. 5, 351-359.

[18] _ New characterizations of input-to-state stability, IEEE Trans. Automat. Control 41 (1996), no. 9, 1283-1294.

[19] A. R. Teel and L. Praly, A smooth Lyapunov function from a class- $\mathcal{K} \mathcal{L}$ estimate involving two positive semidefinite functions, ESAIM Control Optim. Calc. Var. 5 (2000), 313-367 (electronic). 